\title{
Positive Solutions for Multipoint Boundary Value Problems for Singular Fractional Differential Equations
}

\author{
Mohamed Jleli, ${ }^{1}$ Erdal Karapinar,, ${ }^{2,3}$ and Bessem Samet ${ }^{1}$ \\ ${ }^{1}$ Department of Mathematics, King Saud University, Riyadh, Saudi Arabia \\ ${ }^{2}$ Department of Mathematics, Attlım University, İncek, 06836 Ankara, Turkey \\ ${ }^{3}$ Nonlinear Analysis and Applied Mathematics Research Group (NAAM), King Abdulaziz University, Jeddah, Saudi Arabia
}

Correspondence should be addressed to Erdal Karapinar; erdalkarapinar@yahoo.com

Received 23 September 2013; Accepted 3 February 2014; Published 5 March 2014

Academic Editor: Andrew Pickering

Copyright (C) 2014 Mohamed Jleli et al. This is an open access article distributed under the Creative Commons Attribution License, which permits unrestricted use, distribution, and reproduction in any medium, provided the original work is properly cited.

A class of nonlinear multipoint boundary value problems for singular fractional differential equations is considered. By means of a coupled fixed point theorem on ordered sets, some results on the existence and uniqueness of positive solutions are obtained.

\section{Introduction}

Fractional calculus is used to formulate different phenomena in physics, biology, medicine, and so forth. For more details on the applications of fractional calculus, we refer the reader to [1-4]. On the other hand, some basic theory for the initial value problems of fractional differential equations involving Riemann-Liouville differential operator has been discussed by Lakshmikantham [5-7], Bai and Lü [8], El-Sayed et al. $[9,10]$, Bai [11, 12], Zhang [13], and so forth.

Very recently, Liang and Zhang [14] considered the following $m$-point boundary value problem:

$$
\begin{aligned}
& D_{0^{+}}^{\alpha} u(t)+f(t, u(t))=0, \quad 0<t<1,2<\alpha \leq 3, \\
& u(0)=u^{\prime}(0)=0, \quad u^{\prime}(1)=\sum_{i=1}^{m-2} \beta_{i} u^{\prime}\left(\xi_{i}\right),
\end{aligned}
$$

where $D_{0^{+}}^{\alpha}$ is the Riemann-Liouville fractional derivative, $0<$ $\xi_{1}<\xi_{2}<\cdots<\xi_{m-2}<1$ satisfies $0<\sum_{i=1}^{m-2} \beta_{i} \xi_{i}^{\alpha-2}<1$, and $f:[0,1] \times[0,+\infty) \rightarrow[0,+\infty)$ is continuous and nondecreasing with respect to the second variable. Under some hypotheses, using a fixed point theorem on ordered sets, the authors established the existence and uniqueness of solution to such problem.
Motivated by the abovementioned work, in this paper, we deal with the following multipoint boundary value problem:

$$
\begin{gathered}
D_{0^{+}}^{\alpha} u(t)+f(t, u(t), u(t))=0, \quad 0<t<1,2<\alpha \leq 3, \\
u(0)=u^{\prime}(0)=0, \quad u^{\prime}(1)=\sum_{i=1}^{m-2} \beta_{i} u^{\prime}\left(\xi_{i}\right),
\end{gathered}
$$

where $f:(0,1] \times[0,+\infty) \times[0,+\infty) \rightarrow[0,+\infty)$ is continuous and $\lim _{t \rightarrow 0^{+}} f(t, \cdot, \cdot)=+\infty(f$ is singular at $t=0)$.

\section{Preliminaries}

The following preliminaries will be useful later.

Definition 1. The Riemann-Liouville fractional derivative of order $\alpha>0$ of a continuous function $\varphi:(0,+\infty) \rightarrow \mathbb{R}$ is given by

$$
D_{0^{+}}^{\alpha} \varphi(t)=\frac{1}{\Gamma(n-\alpha)}\left(\frac{d}{d t}\right)^{(n)} \int_{0}^{t} \frac{\varphi(s)}{(t-s)^{\alpha-n+1}} d s,
$$

where $n=[\alpha]+1,[\alpha]$ denotes the integer part of number $\alpha$, provided that the right side is pointwise defined on $(0,+\infty)$. Here, $\Gamma$ is the Euler gamma function defined by

$$
\Gamma(\alpha)=\int_{0}^{+\infty} t^{\alpha-1} e^{-t} d t .
$$


Definition 2. The Riemann-Liouville fractional integral of order $\alpha>0$ of a given function $\varphi:(0,+\infty) \rightarrow \mathbb{R}$ is defined by

$$
I_{0^{+}}^{\alpha} \varphi(t)=\frac{1}{\Gamma(\alpha)} \int_{0}^{t}(t-s)^{\alpha-1} \varphi(s) d s,
$$

provided that the right side is defined on $(0,+\infty)$.

From the definition of the Riemann-Liouville derivative, we can obtain the following statement.

Lemma 3 (see [15]). Let $\alpha>0$. If one assumes that $u \in$ $C(0,1) \cap L(0,1)$, then the fractional differential equation

$$
D_{0^{+}}^{\alpha} u(t)=0
$$

has $u(t)=c_{1} t^{\alpha-1}+c_{2} t^{\alpha-2}+\cdots+c_{N} t^{\alpha-N}, c_{i} \in \mathbb{R}, i=1,2, \ldots, N$ as unique solutions, where $N$ is the smallest integer greater than or equal to $\alpha$.

Lemma 4 (see [15]). Assume that $u \in C(0,1) \cap L(0,1)$ with a fractional derivative of order $\alpha>0$ that belongs to $C(0,1) \cap$ $L(0,1)$. Then

$$
I_{0^{+}}^{\alpha} D_{0^{+}}^{\alpha} u(t)=u(t)+c_{1} t^{\alpha-1}+c_{2} t^{\alpha-2}+\cdots+c_{N} t^{\alpha-N},
$$

for some $c_{i} \in \mathbb{R}, i=1,2, \ldots, N$, where $N$ is the smallest integer greater than or equal to $\alpha$.

Lemma 5 (see [14]). Assume that $\sum_{i=1}^{m-2} \beta_{i} \xi_{i}^{\alpha-2} \neq 1$. If $h \in$ $C([0,1])$, then the boundary value problem

$$
\begin{aligned}
& D_{0^{+}}^{\alpha} u(t)+h(t)=0, \quad 0<t<1,2<\alpha \leq 3, \\
& u(0)=u^{\prime}(0)=0, \quad u^{\prime}(1)=\sum_{i=1}^{m-2} \beta_{i} u^{\prime}\left(\xi_{i}\right),
\end{aligned}
$$

has a unique solution

$$
\begin{aligned}
u(t)= & \int_{0}^{1} G(t, s) h(s) d s+\frac{t^{\alpha-1}}{(\alpha-1)\left(1-\sum_{i=1}^{m-2} \beta_{i} \xi_{i}^{\alpha-2}\right)} \\
& \times \sum_{i=1}^{m-2} \beta_{i} \int_{0}^{1} H\left(\xi_{i}, s\right) h(s) d s,
\end{aligned}
$$

where

$$
G(t, s)= \begin{cases}\frac{t^{\alpha-1}(1-s)^{\alpha-2}-(t-s)^{\alpha-1}}{\Gamma(\alpha)}, & \text { if } 0 \leq s \leq t \leq 1, \\ \frac{t^{\alpha-1}(1-s)^{\alpha-2}}{\Gamma(\alpha)}, & \text { if } 0 \leq t \leq s \leq 1, \\ H(t, s)=\frac{\partial G(t, s)}{\partial t} . & \end{cases}
$$

It is clear that, for all $0 \leq t \leq s \leq 1$, the function $H(t, s) \geq$ 0 . For $0 \leq s \leq t \leq 1$, we have $t s \leq s$; then $t^{\alpha-2}(1-s)^{\alpha-2}-$ $(t-s)^{\alpha-2} \geq 0$. Hence for $0 \leq t \leq s \leq 1$ one has $H(t, s) \geq 0$. The following properties of $G$ will be used later.
Lemma 6 (see [14]). The following properties hold.

(i) $G$ is a nonnegative continuous function on $[0,1] \times$ $[0,1]$;

(ii) $G(\cdot, s)$ is strictly increasing for all $s \in[0,1]$.

Let $(X, \preceq)$ be a partially ordered set endowed with a metric $d$. Let $F: X \times X \rightarrow X$ be a given mapping.

Definition 7 . One says that $(X, \preceq)$ is directed if, for every $(x, y) \in X \times X$, there exists $z \in X$ such that $x \preceq z, y \preceq z$ and there exists $w \in X$ such that $x \geq w, y \geq w$.

Definition 8 . We say that $(X, \preceq, d)$ is regular if the following conditions hold.

$\left(c_{1}\right)$ if $\left\{x_{n}\right\}$ is a nondecreasing sequence in $X$ such that $x_{n} \rightarrow x \in X$, then $x_{n} \preceq x$ for all $n$;

$\left(c_{2}\right)$ if $\left\{y_{n}\right\}$ is a decreasing sequence in $X$ such that $y_{n} \rightarrow$ $y \in X$, then $y_{n} \geq y$ for all $n$.

Example 9. Let $X=C([0, T]), T>0$ be the set of real continuous functions on $[0, T]$. We endow $X$ with the standard metric $d$ given by

$$
d(u, v)=\max _{0 \leq t \leq T}|u(t)-v(t)|, \quad u, v \in X .
$$

We define the partial order $\preceq$ on $X$ by

$$
u, v \in X, \quad u \preceq v \Longleftrightarrow u(t) \leq v(t) \quad \forall t \in[0, T] .
$$

Let $x, y \in X$. For $z=\max \{x, y\}$, that is, $z(t)=$ $\max \{x(t), y(t)\}$, for all $t \in[0, T]$, we have $x \preceq z$ and $y \preceq z$. For $w=\min \{x, y\}$, that is, $w(t)=\min \{x(t), y(t)\}$, for all $t \in[0, T]$, we have $x \geq w$ and $y \geq w$. This implies that $(X, \preceq)$ is directed. Now, let $\left\{x_{n}\right\}$ be a nondecreasing sequence in $X$ such that $d\left(x_{n}, x\right) \rightarrow 0$ as $n \rightarrow \infty$, for some $x \in X$. Then, for all $t \in[0, T],\left\{x_{n}(t)\right\}$ is a nondecreasing sequence of real numbers converging to $x(t)$. Thus, we have $x_{n}(t) \leq x(t)$, for all $n$, that is, $x_{n} \preceq x$ for all $n$. Similarly, if $\left\{y_{n}\right\}$ is a decreasing sequence in $X$ such that $d\left(y_{n}, y\right) \rightarrow 0$ as $n \rightarrow \infty$, for some $y \in X$, we get that $y_{n} \geq y$ for all $n$. Then, we proved that $(X, \preceq, d)$ is regular.

Definition 10 (see [16]). An element $(x, y) \in X \times X$ is called a coupled fixed point of $F$ if

$$
F(x, y)=x, \quad F(y, x)=y .
$$

Definition 11 (see [16]). One says that $F$ has the mixed monotone property if

$$
(x, y),(u, v) \in X \times X, x \preceq u, y \geq v \Longrightarrow F(x, y) \preceq F(u, v) .
$$

Denote by $\Phi$ the set of functions $\varphi:[0,+\infty) \rightarrow[0,+\infty)$ satisfying the following.

$\left(\Phi_{1}\right) \varphi$ is continuous;

$\left(\Phi_{2}\right) \varphi$ is nondecreasing;

$\left(\Phi_{3}\right) \varphi^{-1}(\{0\})=\{0\}$. 
The following two lemmas are fundamental for the proof of our main result.

Lemma 12 (see [17]). Let $(X, \preceq)$ be a partially ordered set and suppose that there exists a metric $d$ on $X$ such that $(X, d)$ is a complete metric space. Let $F: X \times X \rightarrow X$ be a mapping having the mixed monotone property on $X$ such that

$$
\begin{aligned}
\psi(d(F(x, y), F(u, v))) \leq & \psi(\max \{d(x, u), d(y, v)\}) \\
& -\varphi(\max \{d(x, u), d(y, v)\}),
\end{aligned}
$$

for all $x, y, u, v \in X$ with $x \geq u$ and $y \preceq v$, where $\psi, \varphi \in \Phi$. Suppose also that $(X, \preceq, d)$ is regular and there exist $x_{0}, y_{0} \in X$ such that

$$
x_{0} \preceq F\left(x_{0}, y_{0}\right), \quad y_{0} \succeq F\left(y_{0}, x_{0}\right) .
$$

Then, $F$ has a coupled fixed point $\left(x^{*}, y^{*}\right) \in X \times X$. Moreover, if $\left\{x_{n}\right\}$ and $\left\{y_{n}\right\}$ are the sequences in $X$ defined by

$$
x_{n+1}=F\left(x_{n}, y_{n}\right), \quad y_{n+1}=F\left(y_{n}, x_{n}\right), \quad n=0,1, \ldots,
$$

then

$$
\lim _{n \rightarrow \infty} d\left(x_{n}, x^{*}\right)=\lim _{n \rightarrow \infty} d\left(y_{n}, y^{*}\right)=0
$$

Lemma 13 (see [17]). Adding to the hypotheses of Lemma 12 the condition $(X, \preceq)$ is directed; one obtains uniqueness of the coupled fixed point. Moreover, one has the equality $x^{*}=y^{*}$.

\section{Main Result}

Let Banach space $E=C([0,1])$ be endowed with the norm $\|u\|_{\infty}=\max _{0 \leq t \leq 1}|u(t)|$. We define the partial order $\preceq$ on $E$ by

$$
u, v \in E, \quad u \preceq v \Longleftrightarrow u(t) \leq v(t) \quad \forall t \in[0,1] .
$$

In Example 9, we proved that $(E, \preceq)$ with the classic metric given by

$$
d(u, v)=\|u-v\|_{\infty}, \quad u, v \in E
$$

satisfies the following properties: $(E, \preceq)$ is directed and $(E, \preceq, d)$ is regular.

Define the closed cone $P \subset E$ by

$$
P=\{u \in E: u \geq 0\},
$$

where 0 denotes the zero function.
Definition 14. One says that $\left(u^{-}, u^{+}\right) \in C([0,1]) \times C([0,1])$ is a coupled lower and upper solution to (2)-(3) if, for all $t \in[0,1]$, one has

$$
\begin{aligned}
u^{-}(t) \leq & \int_{0}^{1} G(t, s) f\left(s, u^{-}(s), u^{+}(s)\right) d s \\
& +\frac{t^{\alpha-1}}{(\alpha-1)\left(1-\sum_{i=1}^{m-2} \beta_{i} \xi_{i}^{\alpha-2}\right)} \\
& \times \sum_{i=1}^{m-2} \beta_{i} \int_{0}^{1} H\left(\xi_{i}, s\right) f\left(s, u^{-}(s), u^{+}(s)\right) d s, \\
u^{+}(t) \geq & \int_{0}^{1} G(t, s) f\left(s, u^{+}(s), u^{-}(s)\right) d s \\
& +\frac{t^{\alpha-1}}{(\alpha-1)\left(1-\sum_{i=1}^{m-2} \beta_{i} \xi_{i}^{\alpha-2}\right)} \\
& \times \sum_{i=1}^{m-2} \beta_{i} \int_{0}^{1} H\left(\xi_{i}, s\right) f\left(s, u^{+}(s), u^{-}(s)\right) d s .
\end{aligned}
$$

The main result of this paper is the following.

Theorem 15. Suppose that the following conditions hold.

(i) $0<\xi_{1}<\xi_{2}<\cdots<\xi_{m-2}<1$ satisfies $0<$ $\sum_{i=1}^{m-2} \beta_{i} \xi_{i}^{\alpha-2}<1$ with $\beta_{i}>0$ for $i=1, \ldots, m-2 ;$

(ii) $f:(0,1] \times[0,+\infty) \times[0,+\infty) \rightarrow[0,+\infty)$ is continuous, $\lim _{t \rightarrow 0^{+}} f(t, \cdot, \cdot)=+\infty$;

(iii) there exists $\sigma \in(0,1)$ such that $t \mapsto t^{\sigma} f(t, x, y)$ is continuous on $[0,1]$ for all $x, y \in[0,+\infty)$;

(iv) there exists

$0<\lambda$

$$
\leq\left[\left(\frac{1-\sum_{i=1}^{m-2} \beta_{i} \xi_{i}^{\alpha-\sigma-1}}{1-\sum_{i=1}^{m-2} \beta_{i} \xi_{i}^{\alpha-2}}-\frac{\alpha-1}{\alpha-\sigma}\right) \frac{\Gamma(1-\sigma)}{(\alpha-1) \Gamma(\alpha-\sigma)}\right]^{-1}
$$

such that for all $x, y, z, w \in[0,+\infty)$ with $x \geq z, y \leq w$ and $t \in[0,1]$,

$$
\begin{aligned}
0 & \leq t^{\sigma}(f(t, x, y)-f(t, z, w)) \\
& \leq \lambda \eta(\max \{(x-z),(w-y)\}),
\end{aligned}
$$

where $\eta:[0,+\infty) \rightarrow[0,+\infty)$ is nondecreasing, $\beta:$ $u \mapsto u-\eta(u) \in \Phi$;

(v) equations (2)-(3) has a coupled lower and upper solution $\left(u^{-}, u^{+}\right) \in P \times P$.

Then,

(I) the boundary value problem (2)-(3) has a unique positive solution $u^{*} \in C([0,1])$; 
(II) the sequences $\left\{u_{n}\right\}$ and $\left\{v_{n}\right\}$ defined by

$$
\begin{aligned}
u_{0}= & u^{-} \\
u_{n+1}= & \int_{0}^{1} G(t, s) f\left(s, u_{n}(s), v_{n}(s)\right) d s \\
& +\frac{t^{\alpha-1}}{(\alpha-1)\left(1-\sum_{i=1}^{m-2} \beta_{i} \xi_{i}^{\alpha-2}\right)} \\
& \times \sum_{i=1}^{m-2} \beta_{i} \int_{0}^{1} H\left(\xi_{i}, s\right) f\left(s, u_{n}(s), v_{n}(s)\right) d s, \\
v_{0}= & u^{+}, \\
v_{n+1}= & \int_{0}^{1} G(t, s) f\left(s, v_{n}(s), u_{n}(s)\right) d s \\
& +\frac{t^{\alpha-1}}{(\alpha-1)\left(1-\sum_{i=1}^{m-2} \beta_{i} \xi_{i}^{\alpha-2}\right)} \\
& \times \sum_{i=1}^{m-2} \beta_{i} \int_{0}^{1} H\left(\xi_{i}, s\right) f\left(s, v_{n}(s), u_{n}(s)\right) d s \\
& \\
& \\
&
\end{aligned}
$$

converge uniformly to $u^{*}$.

Proof. Suppose that $u$ is a solution to the boundary value problem (2)-(3). Then, from Lemma 5, we have

$$
\begin{aligned}
u(t)= & \int_{0}^{1} G(t, s) f(s, u(s), u(s)) d s \\
& +\frac{t^{\alpha-1}}{(\alpha-1)\left(1-\sum_{i=1}^{m-2} \beta_{i} \xi_{i}^{\alpha-2}\right)} \\
& \times \sum_{i=1}^{m-2} \beta_{i} \int_{0}^{1} H\left(\xi_{i}, s\right) f(s, u(s), u(s)) d s,
\end{aligned}
$$

for all $t \in[0,1]$.

Consider the operator $F$ defined by

$$
\begin{aligned}
F(u, v)(t)= & \int_{0}^{1} G(t, s) f(s, u(s), v(s)) d s \\
& +\frac{t^{\alpha-1}}{(\alpha-1)\left(1-\sum_{i=1}^{m-2} \beta_{i} \xi_{i}^{\alpha-2}\right)} \\
& \times \sum_{i=1}^{m-2} \beta_{i} \int_{0}^{1} H\left(\xi_{i}, s\right) f(s, u(s), v(s)) d s
\end{aligned}
$$

for all $t \in[0,1]$, for all $u, v \in P$. From (iii) and Lemma 6, we have that $F(P \times P) \subset P$.
Let $(x, y),(u, v) \in P \times P$ such that $x \preceq u$ and $y \succeq v$. From (25), we have

$$
s^{\sigma} f(s, x(s), y(s)) \leq s^{\sigma} f(s, u(s), v(s)), \quad \forall s \in[0,1] .
$$

Since the operator $F$ is linear and increasing with respect to the function $f$, we deduce that

$$
F(x, y)(t) \leq F(u, v)(t), \quad \forall s \in[0,1]
$$

This implies that $F$ has the mixed monotone property with respect to the partial order $\preceq$ given by $(20)$.

In the sequel, we denote

$$
\xi=\sum_{i=1}^{m-2} \beta_{i} \xi_{i}^{\alpha-2}
$$

Let $(x, y),(u, v) \in P \times P$ such that $x \geq u$ and $y \preceq v$. For all $t \in[0,1]$, using (25) and Lemma 6 , we have

$$
\begin{aligned}
& |F(x, y)(t)-F(u, v)(t)| \\
& =\int_{0}^{1} G(t, s)[f(s, x(s), y(s))-f(s, u(s), v(s))] d s \\
& +\frac{t^{\alpha-1}}{(\alpha-1)(1-\xi)} \\
& \times \sum_{i=1}^{m-2} \beta_{i} \int_{0}^{1} H\left(\xi_{i}, s\right)[f(s, x(s), y(s)) \\
& -f(s, u(s), v(s))] d s \\
& =\int_{0}^{1} G(t, s) s^{-\sigma} s^{\sigma}[f(s, x(s), y(s))-f(s, u(s), v(s))] d s \\
& +\frac{t^{\alpha-1}}{(\alpha-1)(1-\xi)} \\
& \times \sum_{i=1}^{m-2} \beta_{i} \int_{0}^{1} H\left(\xi_{i}, s\right) s^{-\sigma} s^{\sigma}[f(s, x(s), y(s)) \\
& -f(s, u(s), v(s))] d s \\
& \leq \int_{0}^{1} G(t, s) s^{-\sigma} \lambda \eta(\max \{x(s)-u(s), v(s)-y(s)\}) d s \\
& +\frac{t^{\alpha-1}}{(\alpha-1)(1-\xi)} \\
& \times \sum_{i=1}^{m-2} \beta_{i} \int_{0}^{1} H\left(\xi_{i}, s\right) s^{-\sigma} \lambda \eta \\
& \times(\max \{x(s)-u(s), v(s)-y(s)\}) d s
\end{aligned}
$$




$$
\begin{aligned}
& \leq \lambda \eta(\max \{d(x, u), d(y, v)\}) \\
& \times \max _{z \in[0,1]}\left(\int_{0}^{1} G(z, s) s^{-\sigma} d s+\frac{z^{\alpha-1}}{(\alpha-1)(1-\xi)}\right. \\
&\left.\quad \times \sum_{i=1}^{m-2} \beta_{i} \int_{0}^{1} H\left(\xi_{i}, s\right) s^{-\sigma} d s\right) .
\end{aligned}
$$

Thus, for all $(x, y),(u, v) \in P \times P$ such that $x \geq u$ and $y \preceq v$, we have

$$
d(F(x, y), F(u, v)) \leq \lambda \chi \eta(\max \{d(x, u), d(y, v)\}),
$$

where

$$
\begin{aligned}
\chi=\max _{z \in[0,1]}\left(\int_{0}^{1} G(z, s) s^{-\sigma} d s+\frac{z^{\alpha-1}}{(\alpha-1)(1-\xi)}\right. \\
\left.\quad \times \sum_{i=1}^{m-2} \beta_{i} \int_{0}^{1} H\left(\xi_{i}, s\right) s^{-\sigma} d s\right)
\end{aligned}
$$

Now, let $z \in[0,1]$. We have

$$
\begin{array}{rl}
\int_{0}^{1} & G(z, s) s^{-\sigma} d s \\
= & \int_{0}^{z} \frac{z^{\alpha-1}(1-s)^{\alpha-2}-(z-s)^{\alpha-1}}{\Gamma(\alpha)} s^{-\sigma} d s \\
& +\int_{z}^{1} \frac{z^{\alpha-1}(1-s)^{\alpha-2}}{\Gamma(\alpha)} s^{-\sigma} d s \\
= & \frac{1}{\Gamma(\alpha)}\left(z^{\alpha-1} \int_{0}^{1}(1-s)^{\alpha-2} s^{-\sigma} d s\right. \\
= & \frac{z^{\alpha-1}}{\Gamma(\alpha)}\left(\int_{0}^{z}(1-s)^{\alpha-2} s^{-\sigma} d s\right. \\
& \left.-z^{1-\sigma} \int_{0}^{1}(1-s)^{\alpha-1} s^{-\sigma} d s\right) \\
= & \frac{z^{\alpha-1}}{\Gamma(\alpha)}\left(B(1-\sigma, \alpha-1)-z^{1-\sigma} B(1-\sigma, \alpha)\right)
\end{array}
$$

where $B$ denotes the beta function. Recall that beta and gamma functions satisfy the following properties:

$$
\begin{aligned}
& B(a, b)=\int_{0}^{1}(1-s)^{b-1} s^{a-1} d s=\frac{\Gamma(a) \Gamma(b)}{\Gamma(a+b)}, \\
& \Gamma(a+1)=a \Gamma(a) \quad \text { for } a, b>0 .
\end{aligned}
$$

Thus we have

$$
\int_{0}^{1} G(z, s) s^{-\sigma} d s=\frac{z^{\alpha-1}}{\Gamma(\alpha)}\left(1-\frac{\alpha-1}{\alpha-\sigma} z^{1-\sigma}\right) B(1-\sigma, \alpha-1) .
$$

Using the same computation as above, we can show that, for all $i=1, \ldots, m-2$, we have

$$
\int_{0}^{1} H\left(\xi_{i}, s\right) s^{-\sigma} d s=\frac{\alpha-1}{\Gamma(\alpha)} \xi_{i}^{\alpha-2}\left(1-\xi_{i}^{1-\sigma}\right) B(1-\sigma, \alpha-1) .
$$

Now, (37) and (38) give us that

$$
\begin{gathered}
\int_{0}^{1} G(z, s) s^{-\sigma} d s+\frac{z^{\alpha-1}}{(\alpha-1)(1-\xi)} \sum_{i=1}^{m-2} \beta_{i} \int_{0}^{1} H\left(\xi_{i}, s\right) s^{-\sigma} d s \\
=\frac{z^{\alpha-1}}{\Gamma(\alpha)}\left(\frac{1-\tilde{\xi}}{1-\xi}-\frac{\alpha-1}{\alpha-\sigma} z^{1-\sigma}\right) B(1-\sigma, \alpha-1),
\end{gathered}
$$

where

$$
\widetilde{\xi}=\sum_{i=1}^{m-2} \beta_{i} \xi_{i}^{\alpha-\sigma-1} \leq \xi
$$

But the maximum of the above function depending in $z$ is attained at $z=1$. Then

$$
\begin{aligned}
\chi & =\left(\frac{1-\tilde{\xi}}{1-\xi}-\frac{\alpha-1}{\alpha-\sigma}\right) \frac{B(1-\sigma, \alpha-1)}{\Gamma(\alpha)} \\
& =\left(\frac{1-\tilde{\xi}}{1-\xi}-\frac{\alpha-1}{\alpha-\sigma}\right) \frac{\Gamma(1-\sigma)}{(\alpha-1) \Gamma(\alpha-\sigma)}
\end{aligned}
$$

Now, it follows from (33), (41), and condition (iv) that

$$
\begin{aligned}
& \psi(d(F(x, y), F(u, v))) \\
& \leq \psi(\max \{d(x, u), d(y, v)\})-\varphi(\max \{d(x, u), d(y, v)\}),
\end{aligned}
$$

with $\psi(t)=t$ and $\varphi \equiv \beta$.

Finally, taking $\left(x_{0}, y_{0}\right)=\left(u^{-}, u^{+}\right) \in P \times P$, we have from condition (v) that $x_{0} \preceq F\left(x_{0}, y_{0}\right)$ and $y_{0} \succeq F\left(y_{0}, x_{0}\right)$.

Now, from Lemmas 12 and 13 , there exists a unique $u^{*} \in P$ such that $u^{*}=F\left(u^{*}, u^{*}\right)$; that is, $u^{*}$ is the unique positive solution to (2)-(3). The convergence of the sequences $\left\{u_{n}\right\}$ and $\left\{v_{n}\right\}$ to $u^{*}$ follows immediately from (19). This makes end to the proof.

Example 16. Consider the fractional boundary value problem

$$
D_{0^{+}}^{5 / 2} u(t)+\frac{(t-1 / 2)^{2}}{2 \sqrt{t}}\left(u(t)+\frac{1}{u(t)+1}\right)=0, \quad 0<t<1,
$$

$$
u(0)=u^{\prime}(0)=0, \quad 2 u^{\prime}(1)=u^{\prime}\left(\frac{1}{4}\right)
$$


In this case, we have

$$
\begin{aligned}
& 2<\alpha=\frac{5}{2}<3, \quad \beta_{1}=\frac{1}{2}, \quad \xi_{1}=\frac{1}{4}, \\
& \xi=\frac{1}{4}, \quad \widetilde{\xi}=\frac{1}{8}, \\
& f(t, x, y)=\frac{(t-1 / 2)^{2}}{2 \sqrt{t}}\left(x+\frac{1}{y+1}\right), \\
& \forall t \in(0,1], x, y \geq 0 .
\end{aligned}
$$

Note that $f$ is continuous on $(0,1] \times[0,+\infty) \times[0,+\infty)$ and $\lim _{t \rightarrow 0^{+}} f(t, \cdot, \cdot)=+\infty$. Let $\sigma=\lambda=1 / 2$ and $\eta(t)=(1 / 2) t$. For all $x, y, z, w \in[0,+\infty)$ with $x \geq z, y \leq w$ and $t \in[0,1]$, we have

$$
\begin{aligned}
0 & \leq t^{1 / 2}(f(t, x, y)-f(t, z, w)) \\
& =\frac{(t-1 / 2)^{2}}{2}\left[(x-z)+\frac{(w-y)}{(y+1)(w+1)}\right] \\
& \leq\left(t-\frac{1}{2}\right)^{2} \max \{x-z, w-y\} \\
& \leq \frac{1}{4} \max \{x-z, w-y\} \\
& =\lambda \eta(\max \{x-z, w-y\}) .
\end{aligned}
$$

On the other hand, we have

$$
\begin{aligned}
0<\lambda & =\frac{1}{2}<\left[\left(\frac{1-\beta_{1} \xi_{1}^{\alpha-\sigma-1}}{1-\beta_{1} \xi_{1}^{\alpha-2}}-\frac{\alpha-1}{\alpha-\sigma}\right) \frac{\Gamma(1-\sigma)}{(\alpha-1) \Gamma(\alpha-\sigma)}\right]^{-1} \\
& =\frac{18}{5 \sqrt{\pi}} .
\end{aligned}
$$

Now, since $G, H$, and $f$ are nonnegative continuous functions, it is easy to prove that $u^{-} \equiv 0$ is a lower solution to (43)-(44). Moreover, for all $c>0$, we use (37), (38), and (41) to obtain

$$
\begin{aligned}
& \int_{0}^{1} G(t, s) f(s, c, 0) d s \\
& +\frac{t^{\alpha-1}}{(\alpha-1)(1-\xi)} \beta_{1} \int_{0}^{1} H\left(\xi_{1}, s\right) f(s, c, 0) d s \\
& \quad \leq \frac{c+1}{8}\left(\int_{0}^{1} G(t, s) s^{-\sigma} d s\right. \\
& \left.\quad \quad+\frac{t^{\alpha-1}}{(\alpha-1)(1-\xi)} \beta_{1} \int_{0}^{1} H\left(\xi_{i}, s\right) s^{-\sigma} d s\right) \\
& \quad=\frac{5 \sqrt{\pi}}{144}\left(\frac{1-\tilde{\xi}}{1-\xi}-\frac{\alpha-1}{\alpha-\sigma}\right) \frac{\Gamma(1-\sigma)}{(\alpha-1) \Gamma(\alpha-\sigma)}
\end{aligned}
$$

Then, an upper solution $u^{+}$to (43)-(44) will be any constant $c>0$ satisfying

$$
\frac{5 \sqrt{\pi}}{144}(c+1) \leq c .
$$

Finally, from Theorem 15, Problems (43) and (44) have a unique positive solution.

\section{Conflict of Interests}

The authors declare that there is no conflict of interests regarding the publication of this paper.

\section{Authors' Contributions}

All authors contributed equally and significantly to writing this paper. All authors read and approved the final manuscript.

\section{Acknowledgments}

This project was supported by King Saud University, Deanship of Scientific Research, College of Science Research Center.

\section{References}

[1] M. Jleli and B. Samet, "On positive solutions for a class of singular nonlinear fractional differential equations," Boundary Value Problems, vol. 2012, article 73, 2012.

[2] A. A. Kilbas, H. M. Srivastava, and J. J. Trujillo, Theory and Applications of Fractional Differential Equations, vol. 204 of NorthHolland Mathematics Studies, Elsevier Science, Amsterdam, The Netherlands, 2006.

[3] G. A. Losa, D. Merlini, T. F. Nonnenmacher, and E. R. Weibel, Eds., Fractals in Biology and Medicine, vol. 2, Birkhäuser, Basel, Switzerland, 1998.

[4] S. G. Samko, A. A. Kilbas, and O. I. Marichev, Fractional Integrals and Derivatives, Gordon and Breach Science Publishers, Yverdon, Switzerland, 1993.

[5] V. Lakshmikantham, "Theory of fractional functional differential equations," Nonlinear Analysis: Theory, Methods \& Applications, vol. 69, no. 10, pp. 3337-3343, 2008.

[6] V. Lakshmikantham and A. S. Vatsala, "Basic theory of fractional differential equations," Nonlinear Analysis: Theory, Methods \& Applications, vol. 69, no. 8, pp. 2677-2682, 2008.

[7] V. Lakshmikantham and A. S. Vatsala, "General uniqueness and monotone iterative technique for fractional differential equations," Applied Mathematics Letters, vol. 21, no. 8, pp. 828-834, 2008.

[8] Z. Bai and H. Lü, "Positive solutions for boundary value problem of nonlinear fractional differential equation," Journal of Mathematical Analysis and Applications, vol. 311, no. 2, pp. 495505, 2005.

[9] A. M. A. El-Sayed, A. E. M. El-Mesiry, and H. A. A. El-Saka, "On the fractional-order logistic equation," Applied Mathematics Letters, vol. 20, no. 7, pp. 817-823, 2007.

[10] M. El-Shahed, "Positive solutions for boundary value problem of nonlinear fractional differential equation," Abstract and Applied Analysis, vol. 2007, Article ID 10368, 8 pages, 2007. 
[11] C. Bai, "Positive solutions for nonlinear fractional differential equations with coefficient that changes sign," Nonlinear Analysis: Theory, Methods \& Applications, vol. 64, no. 4, pp. 677-685, 2006.

[12] C. Bai, "Triple positive solutions for a boundary value problem of nonlinear fractional differential equation," Electronic Journal of Qualitative Theory of Differential Equations, no. 24, pp. 1-10, 2008.

[13] S. Zhang, "Existence of solution for a boundary value problem of fractional order," Acta Mathematica Scientia B, vol. 26, no. 2, pp. 220-228, 2006.

[14] S. Liang and J. Zhang, "Existence and uniqueness of positive solutions to $m$-point boundary value problem for nonlinear fractional differential equation," Journal of Applied Mathematics and Computing, vol. 38, no. 1-2, pp. 225-241, 2012.

[15] X. Xu, D. Jiang, and C. Yuan, "Multiple positive solutions for the boundary value problem of a nonlinear fractional differential equation," Nonlinear Analysis: Theory, Methods \& Applications, vol. 71, no. 10, pp. 4676-4688, 2009.

[16] T. Gnana Bhaskar and V. Lakshmikantham, "Fixed point theorems in partially ordered metric spaces and applications," Nonlinear Analysis: Theory, Methods \& Applications, vol. 65, no. 7, pp. 1379-1393, 2006.

[17] J. Harjani, B. López, and K. Sadarangani, "Fixed point theorems for mixed monotone operators and applications to integral equations," Nonlinear Analysis: Theory, Methods \& Applications, vol. 74, no. 5, pp. 1749-1760, 2011. 


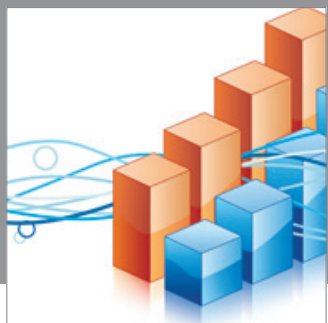

Advances in

Operations Research

mansans

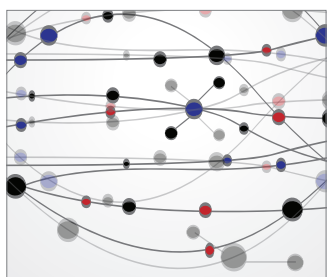

The Scientific World Journal
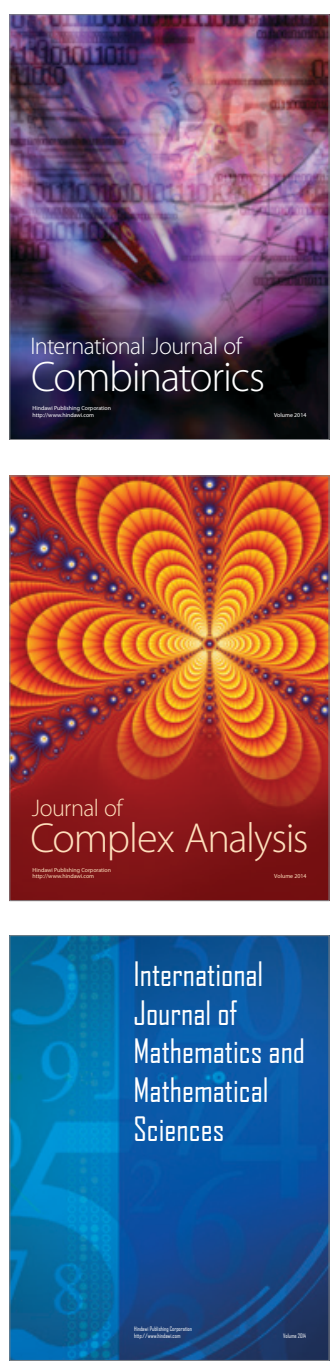
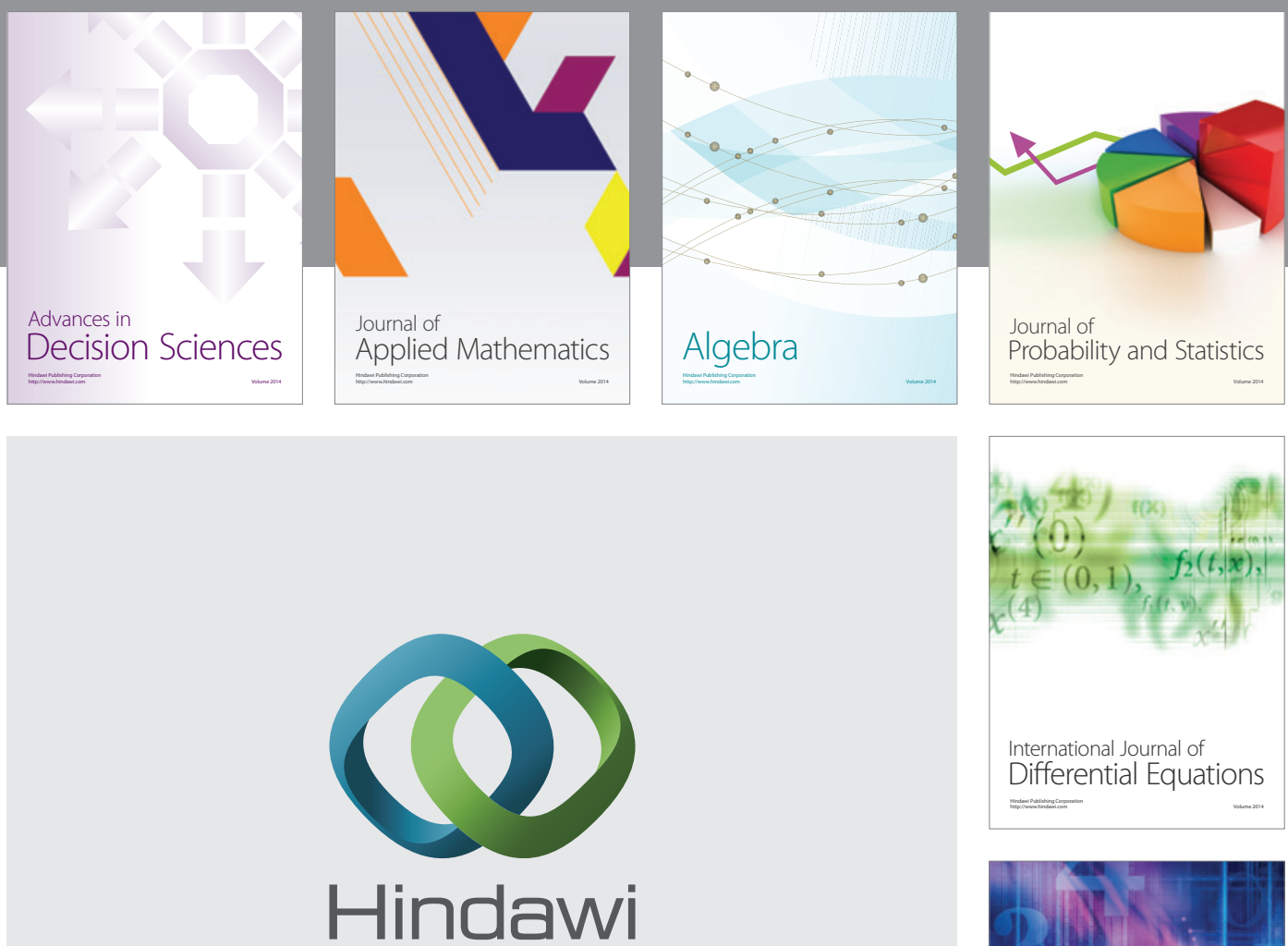

Submit your manuscripts at http://www.hindawi.com
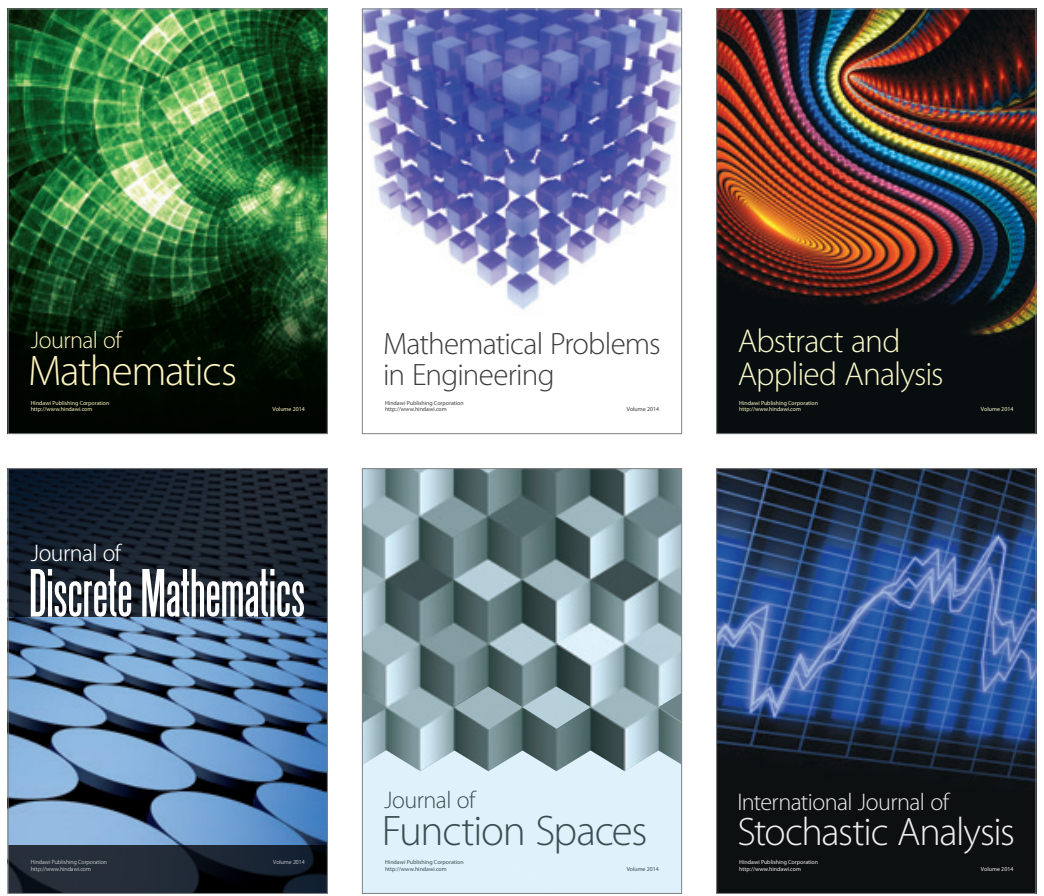

Journal of

Function Spaces

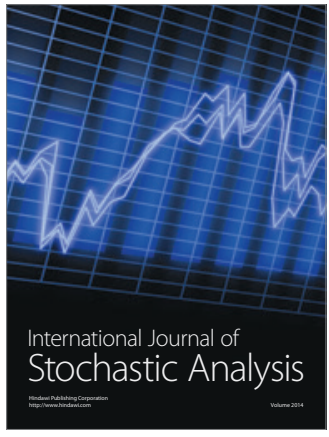

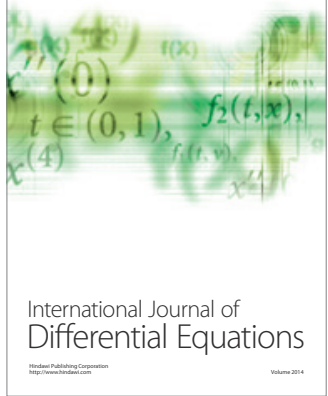
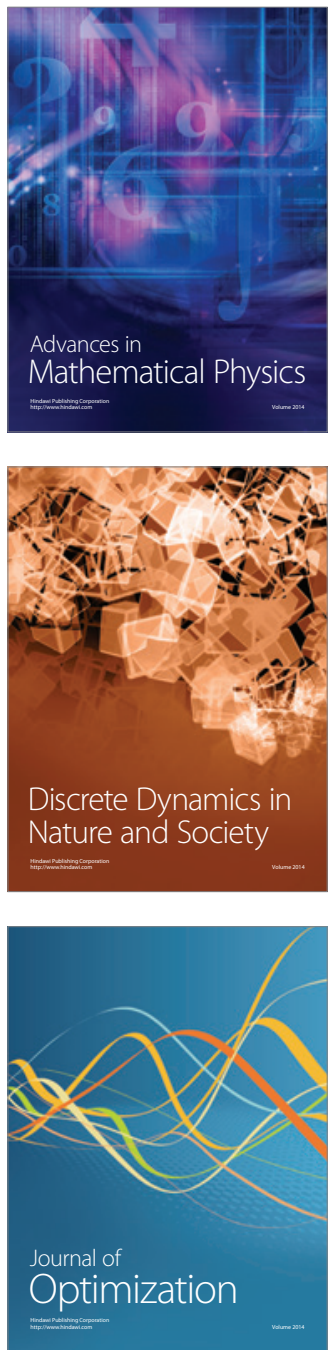